\title{
Wann ist es eine Glutensensitivität?
}

D ie nicht Zöliakie-bedingte Glutenunverträglichkeit, häufig auch als Glutensensitivität bezeichnet, bekommt seit Kurzem bei der Differenzialdiagnose der Zöliakie immer mehr Beachtung. Von der Glutensensitivität wird vermutet, dass sie eine angeborene Immunantwort auf Weizenproteine ist. Deshalb findet Prof. Dr. Sibylle Koletzko aus München, dass der Name „Weizenintoleranz" für die Erkrankung eigentlich der bessere wäre. Sie wird laut Koletzko derzeit massiv überdiagnostiziert, vor allem durch Selbstdiagnosen. Die Glutensensitivität ist laut Definition keine Krankheit, sondern ein Syndrom. Die Patienten reagieren mit verschiedenen Symptomen, allen voran mit Blähungen, Bauchschmerzen, Diarrhö, aber auch mit extraintestinalen Beschwerden wie Unwohlsein und Müdigkeit.

In einer australischen Studie wurde nicht das Gluten, sondern nicht absorbierbare Kohlenhydrate als Auslöser für die Symptome der nicht Zöliakie-bedingten Glutenunverträglichkeit identifiziert. Diese Kohlenhydrate werden auch FODMAP genannt, was die Abkür- zung für fermentierbare Oligo-, Di- und Monosaccharide sowie Polyole ist. Dazu zählen etwa Laktose, Fruktose und Fruktane. Glutenfreie Produkte enthalten deutlich weniger dieser FODMAP. „Es gibt Menschen, die auf die FODMAP mit einem Reizdarm-Syndrom reagieren." Was die Diagnostik der Erkrankung besonders schwierig macht, sind die subjektiven Symptome sowie ein hoher Placebo- und Nocebo-Effekt. Außerdem wird eine Diät häufig begonnen, bevor eine Zöliakie abgeklärt wurde. Auch die Abgrenzung zur Weizenallergie ist kompliziert. Denn die Weizenallergie kann nicht immer durch das Fehlen eines spezifischen IgE ausgeschlossen und dem Nachweis von IgE bewiesen werden.

Bei Verdacht auf eine nicht Zöliakiebedingte Glutenunverträglichkeit schlägt Koletzko bei Kindern folgendes Vorgehen vor: Zunächst muss eine Zöliakie unter Normalkost serologisch ausgeschlossen werden. Danach schließt sich eine Eliminierung in Form einer FODMAP-Diät an. Bleiben die Beschwerden bestehen, wird auch das Gluten in der Ernährung gemie-

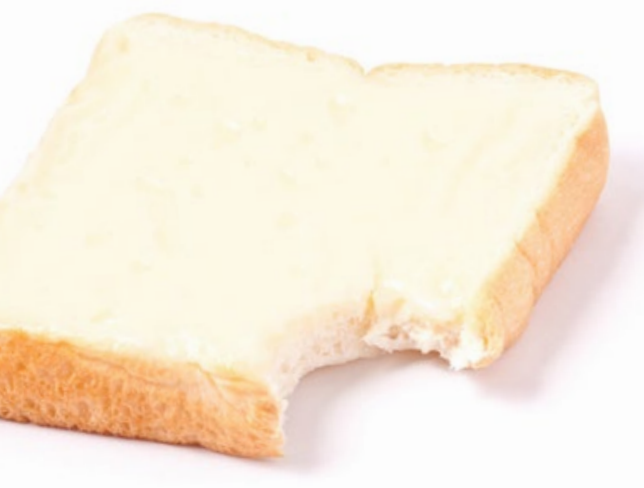

den. Die Patienten sollen außerdem ein Ernährungstagebuch schreiben. Bessern sich die Symptome, schließt sich ein Provokationstest an, der doppelblind durchgeführt wird, da der Nozebo-Effekt sehr $\underset{\oplus}{\varpi}$ hoch ist. Wenn eine Provokation der Überprüfung standhält, dann kommt der Arzt laut Koletzko „in die Nähe einer Diagnose“. Da glutensensitive Patienten teilweise geringe Mengen tolerieren, wird anschließend die Toleranz-Dosis ausgetestet. „Es handelt sich um ein sehr aufwendiges Verfahren und am Ende des Tages bleiben nur wenige Patienten übrig“, fasste Koletzko zusammen.

$n z$

Koletzko S. DD Zöliakie - nicht-Zöliakie-bedingte Glutenunverträglichkeit

\section{Vitamin D: Zufuhrempfehlungen werden nicht erreicht}

$B^{3}$ ei einem Wert unter $50 \mathrm{nmol} / \mathrm{L}$ spricht die Deutsche Gesellschaft für Kinder- und Jugendmedizin von einem

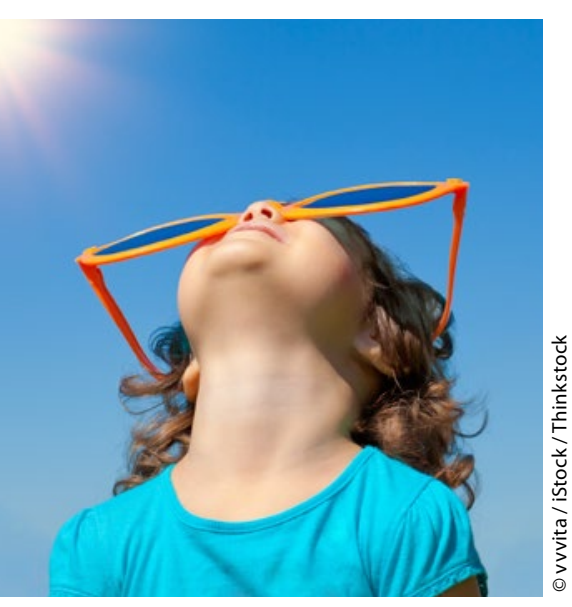

klinisch relevanten Vitamin-D-Mangel. Untersuchungen des Robert-Koch-Instituts und des FKE Dortmund ergaben, dass $15-17 \%$ aller Kinder in Deutschland Werte unter $25 \mathrm{nmol} / \mathrm{L}$ aufweisen, was einem schwerer Vitamin-D-Mangel entspricht. Bei $45 \%$ der Kinder über 3 Jahren kommen Werte zwischen 25 und $50 \mathrm{nmol} / \mathrm{L}$ vor. „Zusammengenommen sind das rund $60-70 \%$ der Kinder, die Probleme mit der Vitamin-D-Versorgung haben“, gab Prof. Dr. Clemens Kunz vom Institut für Ernährungswissenschaft der Justus-Liebig-Universität Gießen zu bedenken.

Die Abdeckung durch Nahrungsmittel sei laut Kunz nur marginal, es werden lediglich 50-100 IE pro Tag aufgenommen. Die Empfehlungen zur Sonnenex- position sind widersprüchlich. Eine Synthese über die Haut ist im Herbst und Winter kaum möglich und in den anderen Monaten nur schlecht abzuschätzen, da sie von vielen Einflussfaktoren abhängt. Deshalb folgert Kunz: „Die fehlenden 500-700 Einheiten Vitamin D können nur über die Aufnahme von Supplementen oder angereicherte Lebensmittel erfolgen." Die Zufuhrempfehlungen liegen bei 600-800 IE.

Die Supplementierung von Vitamin D ist sicher, eine Intoxikation kaum möglich. Die Europäische Behörde für Lebensmittelsicherheit und das US-amerikanische Institute of Medicine legten als sichere tägliche Einnahmemengen für Säuglinge 1.000 IE, Kinder von 1-11 Jahren 2.000 IE und Kinder ab 11 Jahren, Jugendliche und Erwachsene 4.000 IE fest.

Kunz C. Vitamin D im Kindes- und Jugendalter 